\title{
A new method to estimate the ages of globular clusters: the case of NGC 3201
}

\author{
A. Calamida ${ }^{1}$, G. Bono ${ }^{2,3}$, P. B. Stetson ${ }^{4}$, M. Dall'Ora ${ }^{5}$, M. Monelli ${ }^{6}$, \\ C.E. Corsi ${ }^{3}$, P. G. Prada Moroni ${ }^{7}$, S. Degl'Innocenti ${ }^{7}$, A. Dotter ${ }^{8}$, C. \\ Brasseur $^{8}$, P. Amico ${ }^{1}$, E. Marchetti ${ }^{1}$, R. Buonanno ${ }^{2}$, A. Di Cecco ${ }^{2}$, S. \\ D'Odorico $^{1}$, I. Ferraro ${ }^{3}$, G. Iannicola ${ }^{3}$, M. Nonino ${ }^{7}$, M. Romaniello ${ }^{1}$, \\ N. Sanna ${ }^{2}$, D. A. Vandenberg ${ }^{8}$, M. Zoccali ${ }^{9}$, and A. Walker ${ }^{10}$ \\ ${ }^{1}$ ESO, Karl-Schwarzschild-Str. 2, D-85748 Garching bei München, Germany \\ email: acalamid@eso.org \\ ${ }^{2}$ Universita' di Roma Tor Vergata, Via della Ricerca Scientifica 1, 00133 Rome, Italy \\ ${ }^{3}$ INAF-Osservatorio Astronomico di Roma, Via Frascati 33, 00040, Monte Porzio Catone, Italy \\ ${ }^{4}$ DAO, HIA-NRC, 5071 W. Saanich Road, Victoria, BC V9E 2E7, Canada \\ ${ }^{5}$ INAF-Osservatorio Astronomico di Capodimonte, Via Moiariello 16, 80131 Napoli, Italy \\ ${ }^{6}$ IAC - Instituto de Astrofisica de Canarias, Calle Via Lactea, E38200 La Laguna, Tenerife, \\ Spain \\ ${ }^{7}$ INAF-Osservatorio Astronomico di Trieste, Via G.B. Tiepolo 11, 40131 Trieste, Italy \\ ${ }^{8}$ UVIC, Victoria, BC V8W 3P6, Canada \\ ${ }^{9}$ Universidad de Concepcion, Departamento de Fisica, Casilla 106-C, Concepcion, Chile \\ ${ }^{10}$ Cerro Totolo Inter-American Observatory, Chile
}

\begin{abstract}
We devised a new method to estimate globular cluster absolute ages by adopting the knee of the bending of the lower main-sequence (MS) in the Near-Infrared (NIR) $J, J-K_{s}$ color-magnitude diagram. The color difference between this feature and the Turn-Off point is strongly correlated to the cluster age. This method is marginally affected by distance and reddening uncertainties, and by the possible occurrence of differential reddening. Furthermore, the knee location does not depend on the cluster age and it is a robust theoretical prediction. We adopted accurate $J, K_{s}$-band photometry collected with both MAD/VLT and SOFI/NTT for the Galactic globular cluster NGC 3201 to identify the location of the knee at $J \sim 19.90 \pm 0.03$ and $J-K_{s} \sim 0.76 \pm 0.02 \mathrm{mag}$. The comparison with different sets of cluster isochrones, transformed adopting different Color-Temperature-Relations (CTRs), shows that the models are slightly redder than the observations for $J>19 \mathrm{mag}$. This difference could be due to the presence of a calibration drift or to a problem of the CTRs in this magnitude range.
\end{abstract}

Keywords. globular clusters: general, globular clusters: individual (NGC 3201)

\section{Introduction}

The absolute age of Galactic Globular Clusters (GGCs) is a crossroad of several astrophysical problems (Vandenberg et al. 1996; Chaboyer 1998; Castellani 1999). This parameter provides: i) a lower limit to the age of the Universe (Buonanno et al. 1998; Stetson et al. 1999; De Angeli et al. 2005); ii) robust constraint on the input physics adopted in stellar evolutionary models (Castellani \& Degl'Innocenti 1999; Vandenberg et al. 2002, 2008; Dotter et al. 2007), and iii) the chronology for the assembly of the halo, the bulge and the disk of the Milky Way (Rosenberg et al. 1999, Zoccali et al. 
2003). However, estimates of GC ages are still hampered by uncertainties affecting both the distance modulus and the reddening (Renzini 1991; Bono et al. 2008), together with uncertainties on the chemical composition (Gratton et al. 2004), on the metallicity scale (Rutledge et al. 1997; Kraft \& Ivans 2003), and on the photometric zero-points (Stetson 2005).

The GGC NGC 3201 presents some interesting features. It is located at relatively short distance $(\mu=13.32 \pm 0.06, E(B-V)=0.30 \pm 0.03$, Piersimoni et al. $2002 ; \mu=13.36 \pm$ $0.06, E(B-V)=0.25 \pm 0.02$, Layden \& Sarajedini 2003; Mazur et al. 2003), and has accurate estimate of both iron $([F e / H]=-1.54 \pm 0.10$ dex, Kraft \& Ivans 2003; Covey et al. 2003) and $\alpha$-elements $([\alpha / F e] \sim 0.2-0.4$, Pritzl et al. 2005). Moreover, NGC 3201 presents a low central density, a large tidal radius $\left(\log \rho_{V}=2.69 L_{\odot} p c^{-3}, r_{t} \sim 28\right.$ arcmin, Harris 2003), and a highly-retrograde orbit (van den Bergh 1993), thus suggesting that it might not be a typical member of the Galactic halo. It has been associated to different Galactic stellar streams (Grillmair 2006; Belokurov et al. 2007), but these associations were ruled out by Casetti-Dinescu et al. (2007), on the basis of its distance on the Galactic plane. The main drawbacks of NGC 3201 are that it is affected by field contamination, presents a relatively high reddening $(E(B-V)=0.25-0.30)$, and is also affected by differential reddening. Owing to the quoted problems we still lack an accurate estimate of the absolute age of NGC 3201 .

\section{Observations and data reduction}

The $B, V, I$-band data considered in this investigation come from the database of original and archival observations which have been collected, reduced and calibrated by $\mathrm{P}$. B. Stetson (see his proceeding in this book). For NGC 3201 we rely on a catalog with 112,238 stars having at least two measurements in each of the three optical bands. The accuracy of the zero-points in the quoted bands is of the order or better than 0.01 mag.

Near-Infrared (NIR) data $\left(J, K_{s}\right)$ were collected in two observing runs with different pointings of the NIR camera SOFI (Field of View, FoV $5 \times 5 \operatorname{arcmin}^{2}$; pixel scale $=0.29 \mathrm{arcsec} / \mathrm{pixel}$ ) available at the New Technology Telescope (NTT; ESO, La Silla). The total exposure time per band are $\sim 10(J)$ and $\sim 40\left(K_{s}\right)$ minutes, and the images cover an area of $\approx 20 \times 18$ arcmin across the cluster center (a more detailed discussion concerning this data set will be given in a forthcoming paper).

These data were supplemented with deep NIR data $\left(J, K_{s}\right)$ collected with the MultiConjugate Adaptive Optics Demonstrator (MAD) available at the Very Large Telescope (VLT; ESO, Paranal). MAD is a prototype instrument performing wide FoV real-time correction for atmospheric turbulence (Marchetti et al. 2006, Gilmozzi \& Spyromilio 2007). MAD is equipped with an infrared imaging camera, CAMCAO, based on a $2048 \times 2048$ pixels Hawaii2 infrared detector with a pixel scale of 0.028 arcsec/pixel for a total FoV of 1 squared arcminute. During the first on-sky demonstration run of MAD, four $1 \times 1$ arcminutes fields were observed in the region located in the S-W corner of NGC 3201. For wavefront sensing, five guide stars with visual magnitude ranging from 11.8 and 12.9 , equally distributed on a circle of 2 arcmin diameter concentric to the field were used, and the Multi-Conjugate Adaptive Optics (MCAO) loop was closed at a correction frequency of $400 \mathrm{~Hz}$. For each pointing we collected three $J$-band and five $K_{s}$-band images of $240 \mathrm{sec}(D I T=10, N D I T=24)$. The seeing during the observations changed from 0.6 to $0.8 \operatorname{arcsec}\left(J\right.$-band) and from 0.8 to $1.3 \operatorname{arcsec}\left(K_{s}\right.$-band). The fullWidth Half Maximum (FWHM) measured on the images ranges from 0.07 to 0.10 arcsec. Details concerning the pre-reduction strategy adopted will be discussed in a forthcoming paper (Dall'Ora et al. 2009, in preparation). 
Photometry on individual images was performed with DAOPHOT $I V /$ ALLSTAR, followed by simultaneous photometry over 358 NIR images with ALLFRAME (Stetson 1994). The instrumental magnitudes were transformed into the 2MASS photometric system adopting a large sample of local standards. We ended-up with a catalog of $\sim 29,000$ stars with at least one measurement in each of the two NIR bands.

\section{Results and discussion}

In order to validate the cluster isochrones we first performed two independent fits using optical bands. The top panel of Fig. 1 shows the comparison, in the $V, B-I$ color-magnitude diagram (CMD), between selected stars and cluster isochrones at fixed chemical compositions and two different ages (Dotter et al. 2008). Theory was transformed into the observational plane using either the Phoenix atmosphere models (red and purple lines, Brott \& Hauschildt 2005) or with the semi-empirical relations by Vandenberg \& Clem (2003, hereinafter VC03, green and violet lines). Data plotted in this figure show, within the errors, that this cluster has an absolute age of $12 \pm 1$ Gyr. The plausibility of the adopted distance modulus and reddening is supported by the good agreement between predicted and Zero-Age-Horizontal-Branch (ZAHB) and HB stars.

As a further test for the theoretical validation we performed a similar comparison, but using different sets of cluster isochrones (Victoria-Regina: Vandenberg et al. 2006; Dartmouth: Dotter et al. 2008; Pisa: Cariulo et al. 2004) constructed by adopting different physical assumptions, but transformed into the observational plane using the same Color-Temperature-Relations (CTRs, Phoenix models). The NIR CMDs present some advantages when compared to optical CMDs: 1) they are less affected by uncertain and differential reddening; 2) stellar isochrones plotted in Fig. 2 show that the MS in the low-mass regime presents a well-defined bending. This feature is mainly caused by Collisional Induced Absorption (CIA) of molecular hydrogen at NIR wavelengths (Saumon et al. 1994). According to theory, the color and the shape of the bending depend on the metal content (see Fig. 2). However, the magnitude and the color of the bending are, at fixed chemical composition, minimally affected by cluster age. This feature offers the unique opportunity to anchor cluster isochrones, and in turn to estimate the absolute age as a color difference between the knee of the bending and the cluster Turn-Off (TO, Fig. 2). The bending is a robust prediction, since the stellar structures, when moving from this region toward lower stellar masses, are minimally affected by uncertainties in the treatment of the convective transport. The convective motions are indeed adiabatic (Saumon \& Marley 2008). We note that a similar feature has already been detected in two other GGCs ( $\omega$ Cen, Pulone et al. 1998; M4, Pulone et al. 1999) and in the Galactic bulge (Zoccali et al. 2000), using deep NIR $(J, H)$ data collected with NICMOS on the Hubble space Telescope.

According to this evidence we took advantage of the deep NIR CMD (Fig. 3) based on images collected with both SOFI and MAD. Data plotted in this figure display a well defined bending along the MS for $J>19.5$ mag. To properly define the bending, the stellar distribution along the MS was smoothed according to a Gaussian Kernel with standard deviations equal to the photometric error in magnitude and in color. We defined the knee of the bend as the reddest point and we found that it is located at $J \sim 19.90 \pm 0.03$ and $J-K_{s} \sim 0.76 \pm 0.02 \mathrm{mag}$ (see the plus sign in Fig. 3 ). We overplotted the same isochrones, for a fixed chemical composition and ages of 11,13 Gyr, that we have validated on the optical CMDs, and we adopted the same distance modulus but a different reddening, $E(B-V)=0.19$. The $\sim 30 \%$ decrease of the adopted 

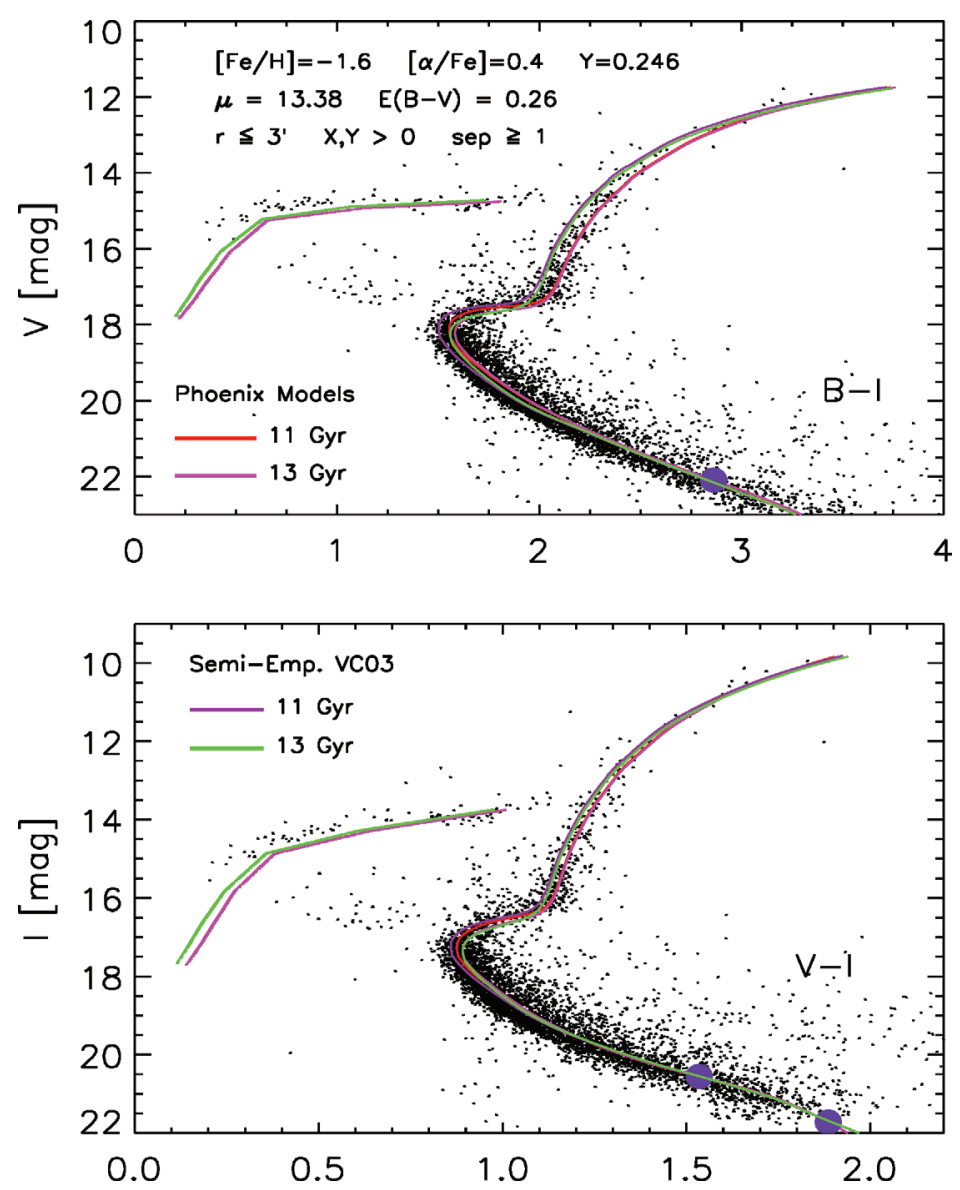

Figure 1. - Top: $V, B-I$ CMD of NGC 3201 based on data collected with ground-based and space telescopes. Stars plotted in this CMD were selected adopting different criteria (separation, position). The red and the purple lines show two cluster isochrones from the Dartmouth database at fixed chemical composition and different ages. These isochrones were transformed into the observational plane using the Phoenix atmosphere models, while the violet and the green isochrones were transformed using the semi-empirical transformation by VC03. The ZAHBs plotted in this CMD have been transformed using the same CTRs. The adopted true distance modulus and cluster reddening are labeled. - Bottom: Same as top, but for the $I, V-I$ CMD. The two large blue circles mark the position of two stellar structures with $M=0.50$ and $0.37 M_{\odot}$, respectively.

reddening in order to fit the NIR CMD could be due to a problem in the reddening law (Cardelli et al. 1989; Fitzpatrick 1999). Moreover, Fig. 3 shows that current isochrones are slightly redder than observations for $J>19$ mag. This could be caused by a drift in the photometric calibration or by a problem with the NIR CTRs in the lower MS regime. However, we need to improve both the accuracy of the photometry and the CTRs in this faint magnitude range.

\section{References}

Belokurov, V., et al. 2007, ApJ, 658, 337

Bono, G., et al. 2008, ApJ, 686, L87 


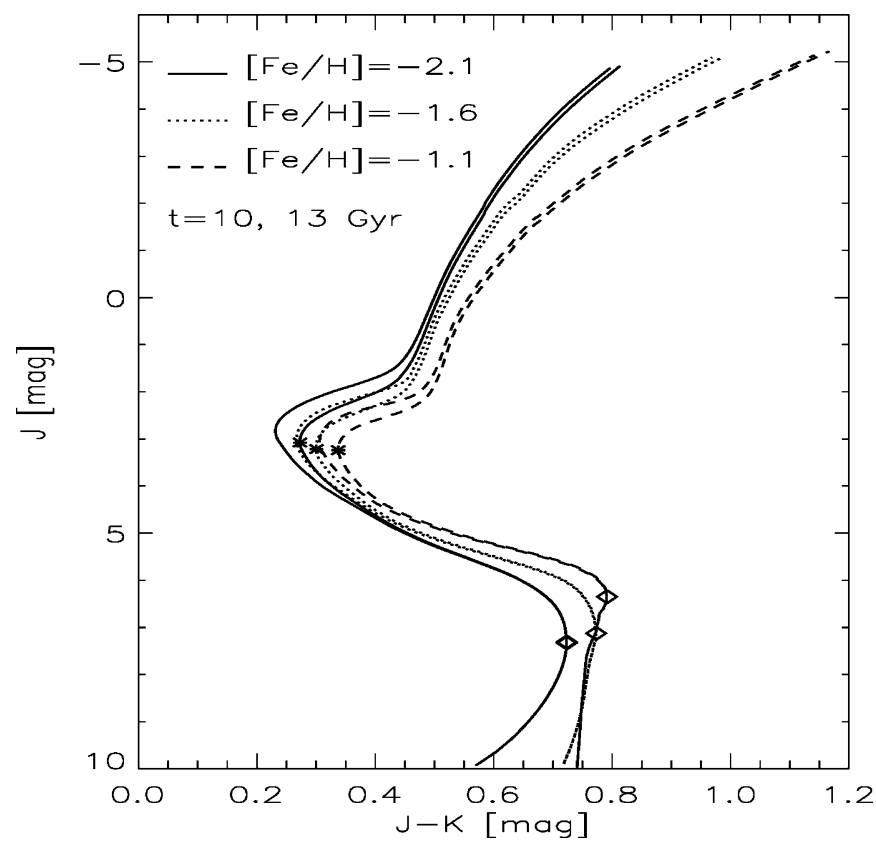

Figure 2. Stellar isochrones from the Dartmouth database with different ages and chemical compositions plotted in the $J, J-K$ CMD. Predictions were transformed into the 2MASS photometric system using Phoenix atmosphere models. The lower MS shows a well defined bending affected by the chemical composition, but independent of cluster age. The asterisks mark the TO position for the isochrones with $t=13$ Gyr, while the diamonds in the lower MS marks the knee of the bending.

Brott, I. \& Hauschildt, P. H. 2005, ESASP, 576, 565

Buonanno, R., et al. 1998, ApJ, 501, L33

Cardelli, J. A., Clayton, \& G. C., Mathis, J. S. 1989, ApJ, 345, 245

Cariulo, P., Degl'Innocenti S., \& Castellani, V. 2004, A\&A 421, 1121

Casetti-Dinescu, D. I., et al. 2007, AJ, 134, 103

Castellani, V. 1999, in Globular clusters, 10th Canary Islands Winter School of Astrophysics, 109

Castellani, V. \& Degl'Innocenti, S. 1999, A\&A, 344, 97

Covey, K. R., et al. 2003, PASP, 115, 819

Chaboyer, B. 1998, PhR, 307, 23

De Angeli, et al. 2005, AJ, 130, 116

Dotter, A., et al. 2007, AJ, 134, 376

Dotter, A., et al. 2008, ApJS, 178, 89

Fitzpatrick, E. L. 1999, PASP, 111, 63

Gilmozzi, R. \& Spyromilio, J. 2007, Msngr, 127, 11

Gratton, R., Sneden, C., \& Carretta, E. 2004, ARA\&A, 42, 385

Grillmair, C. J. 2006, ApJ, 651, 29

Harris, W. E. 2003, Catalog of Parameters for Milky Way Globular Clusters: The Database Hamilton: McMaster Univ., http://physun.physics.mcmaster.ca/ harris/mwgc.dat

Kraft, R. \& Ivans, I. 2003, PASP, 115, 143

Layden, A. C. \& Sarajedini, A. 2003, AJ, 125, 208

Mazur, B., Krzemiski, W., \& Thompson, I. B. 2003, MNRAS, 340, 1205

Marchetti, E., et al. 2006, SPIE, 6272, 21

Piersimoni, A., Bono, G., \& Ripepi, V. 2002, AJ, 124, 1528 
A. Calamida et al.

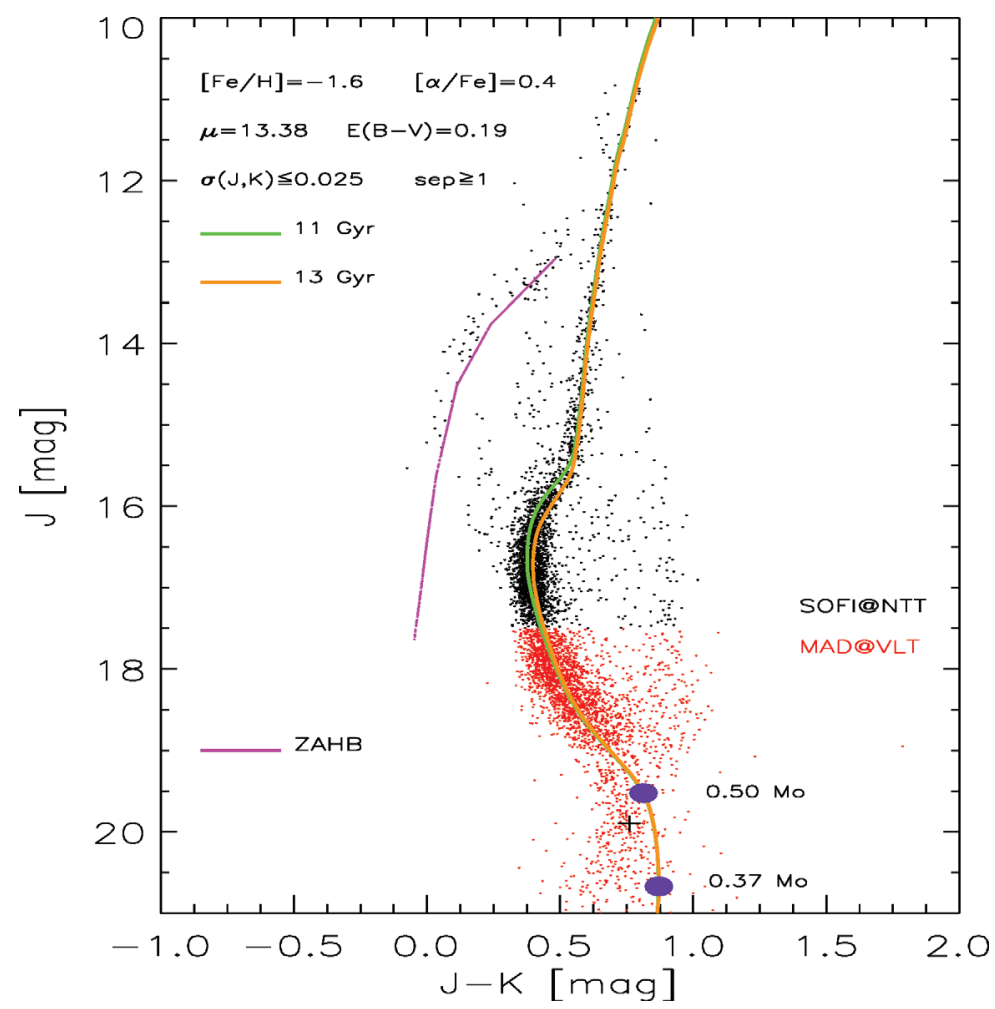

Figure 3. NIR $J, J-K$ CMD of NGC 3201. Black and red dots display stars observed with SOFI/NTT and with MAD/VLT. The green and the yellow lines show two cluster isochrones from the Dartmouth database at fixed chemical composition. The purple line shows the ZAHB for the same chemical composition. The adopted distance modulus and reddening are labeled. The two large blue circles mark the position of two stellar structures with $M=0.50$ and $0.37 M_{\odot}$, respectively.

Pulone, L. et al. 1998, ApJ, 492, 41

Pulone, L., De Marchi, G., \& Paresce, F. 1999, A\&A, 342, 440

Pritzl, B. J., Venn, K. A., \& Irwin, M. 2005, AJ, 130, 2140

Renzini, A. 1991, otci.conf., 131

Rosenberg, A., et al. 1999, AJ, 118, 230

Rutledge, et al. 1997, PASP, 109, 907

Saumon, D., et al. 1994, ApJ, 424, 333

Saumon, D. \& Marley, M. S. 2008, ApJ, 689, 1327

Stetson, P. B. 1994, PASP, 106, 250

Stetson, P. B., et al. 1999, AJ, 117, 247

Stetson, P. B. 2005, PASP, 117, 563, http://www1.cadc-ccda.hia-iha.nrc-cnrc.gc.ca/ community/STETSON/standards

Vandenberg, D., Stetson, P. B., \& Bolte, M. 1996, ARA\&A, 34, 461

Vandenberg, D., et al. 2002, ApJ, 571, 487

Vandenberg, D. \& Clem, J. L. 2003, AJ, 126, 778

Vandenberg, D., et al. 2008, ApJ, 675, 746

van den Bergh, S. 1993, AJ, 105, 971

Zoccali, M., et al. 2000, ApJ, 538, 289

Zoccali, M., et al. 2003, A\& A, 399, 931 


\section{Discussion}

A. SARAJEDINI: Why do you refer to your method as an absolute age indicator? You rely on color differences which depend sensitively on the model colors. Getting an absolute age from these models is especially uncertain.

A. Calamida: We can anchor the isochrones adopting two different reference points, in the near-infrared color-magnitude diagram: the turn-off point that depends on the cluster age and the "knee" of the lower main sequence bending that does not depend on cluster age (see Fig. 2 of the text). Moreover, the bending is a robust theoretical prediction since stellar structure in this region is minimally affected by uncertainties in the treatment of convective transport (see text for details). More importantly, this method is not affected by the uncertainties in the estimate of the cluster distance modulus and by the possible occurrence of differential reddening. We also adopted different sets of cluster isochrones computed in Pisa (Cariulo et al. 2004) and in Victoria (VandenBerg et al. 2006) and different color-temperature relations (Phoenix and the semi-empirical ones of VandenBerg \& Clem 2003) for our near-IR CMD fits. They are in agreement within the uncertainties. This method is much more robust that the classical isochrone fits that rely only on the color and the magnitude of the turn-off point, which is affected by the uncertainties in the cluster distance, reddening, and differential reddening.

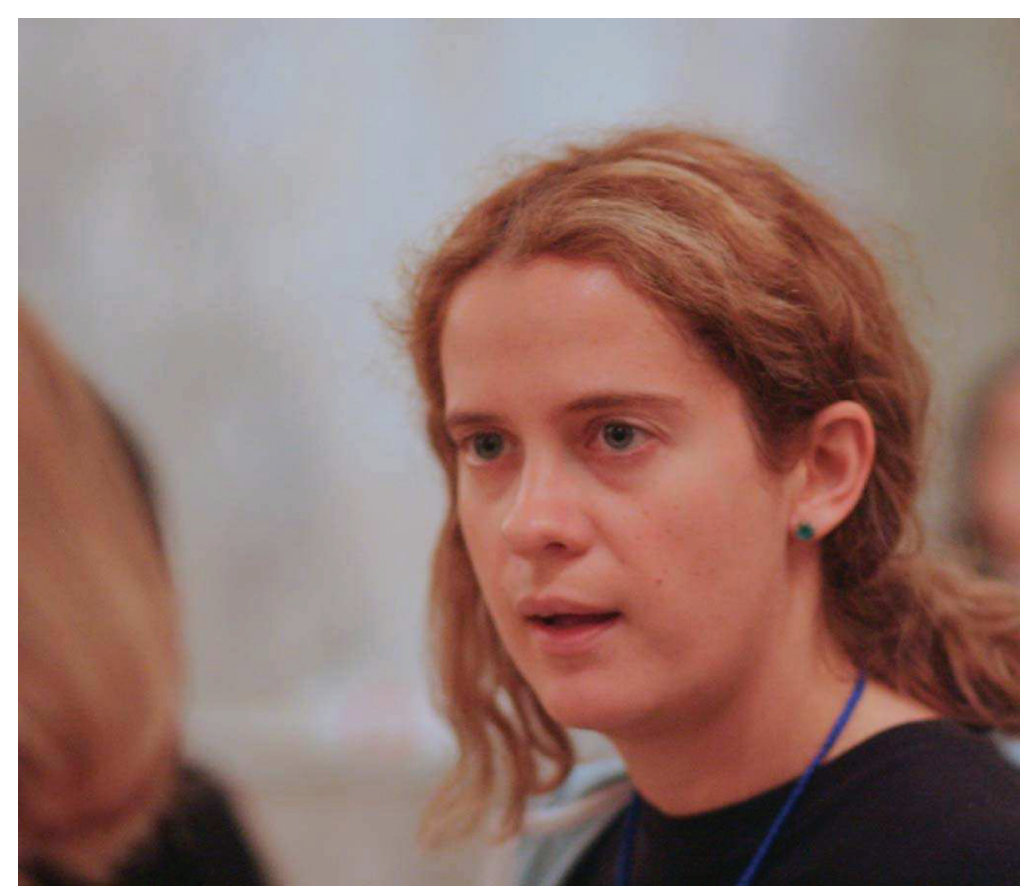

Annalisa Calamida 
A. Calamida et al.

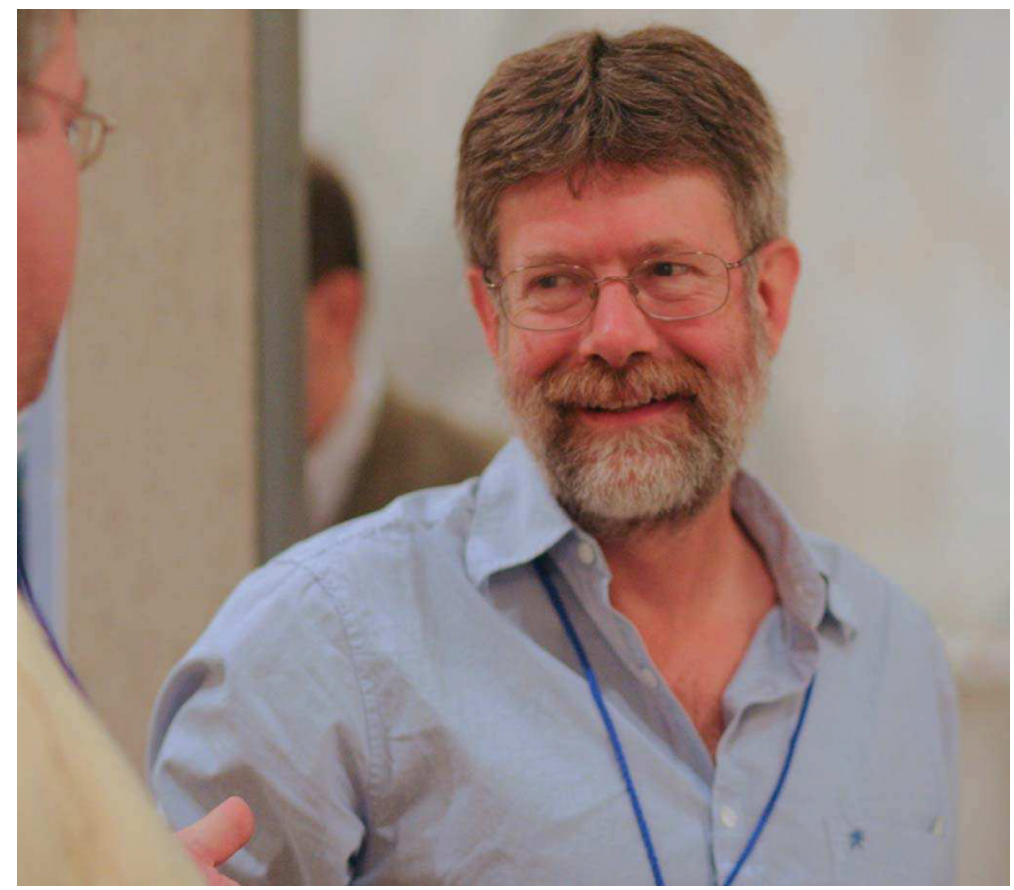

Fred Walter

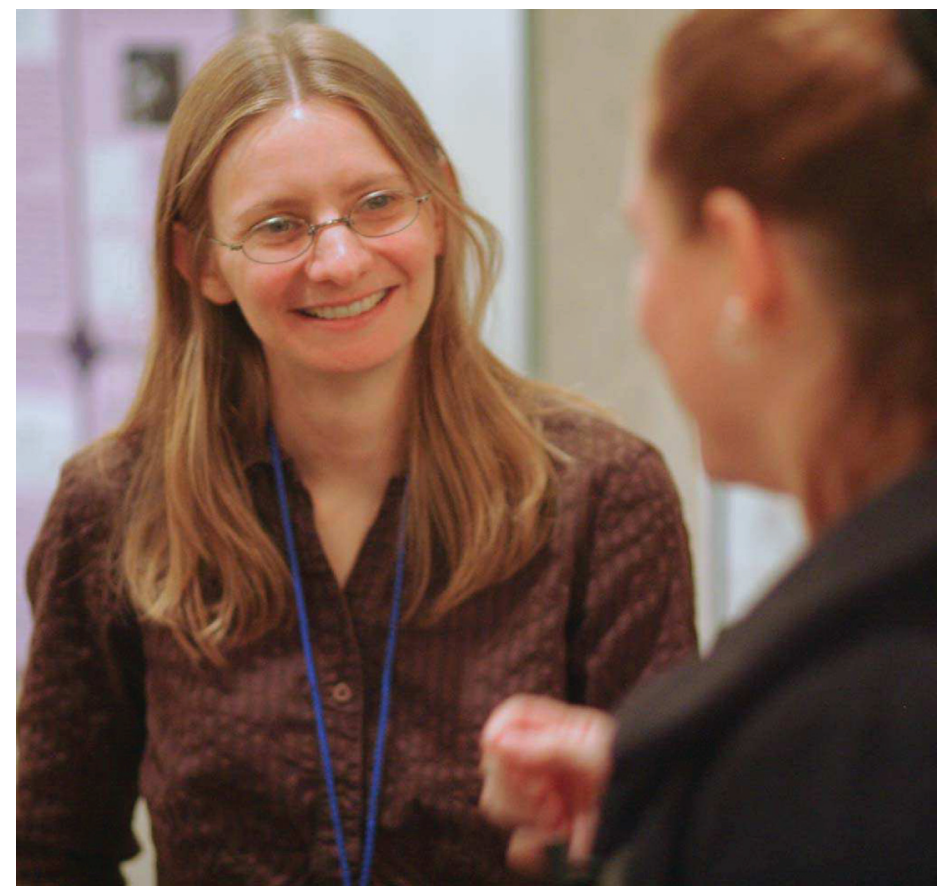

Gail Schaefer 